\title{
8
}
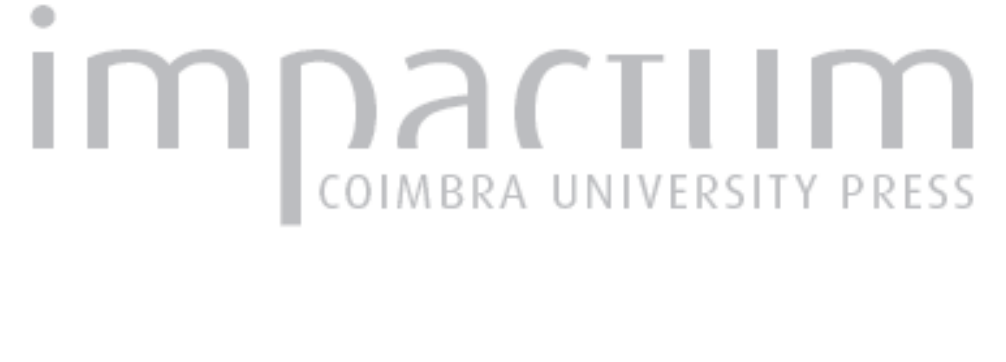

\section{Soldats et cultes indigènes dans les provinces occidentales au Haut-Empire}

Autor(es): Le Roux, Patrick

Publicado por: Faculdade de Letras da Universidade de Coimbra

URL persistente:

URI:http://hdl.handle.net/10316.2/37666

DOI:

DOI:http://dx.doi.org/10.14195/1647-8657_41_4

Accessed : $\quad$ 26-Apr-2023 15:29:25

A navegação consulta e descarregamento dos títulos inseridos nas Bibliotecas Digitais UC Digitalis, UC Pombalina e UC Impactum, pressupõem a aceitação plena e sem reservas dos Termos e Condições de Uso destas Bibliotecas Digitais, disponíveis em https://digitalis.uc.pt/pt-pt/termos.

Conforme exposto nos referidos Termos e Condições de Uso, o descarregamento de títulos de acesso restrito requer uma licença válida de autorização devendo o utilizador aceder ao(s) documento(s) a partir de um endereço de IP da instituição detentora da supramencionada licença.

Ao utilizador é apenas permitido o descarregamento para uso pessoal, pelo que o emprego do(s) título(s) descarregado(s) para outro fim, designadamente comercial, carece de autorização do respetivo autor ou editor da obra.

Na medida em que todas as obras da UC Digitalis se encontram protegidas pelo Código do Direito de Autor e Direitos Conexos e demais legislação aplicável, toda a cópia, parcial ou total, deste documento, nos casos em que é legalmente admitida, deverá conter ou fazer-se acompanhar por este aviso.

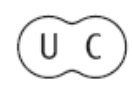




\section{CONIMBRIGA}

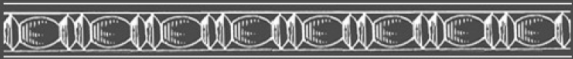

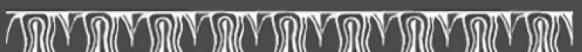
2

INSTITUTO DE ARQUEOLOGIA

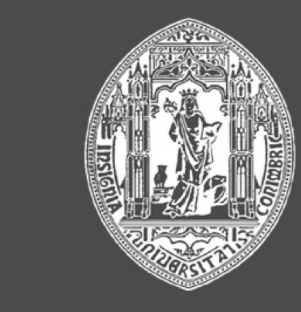

VOLUME XLI - 2002

F A C U L D A E D E LETRAS UNIVERSIDADE DE COIMBRA 
PATRICK Le RouX

L'Année Épigraphique. Paris

\section{SOLDATS ET CULTES INDIGÈNES DANS LES PROVINCES OCCIDENTALES AU HAUT-EMPIRE \\ "Conimbriga" XLI (2002) p. 105-126}

RÉSUMÉ: L'article traite des questions de méthode soulevées par l'interprétation de diverses inscriptions religieuses des provinces occidentales, à caractère personnel, faisant suite à l'accomplissement d' un vœu par des soldats ou des officiers en service, en l'absence d'un contexte bien défini. On prête tout particulièrement attention aux dévotions envers les divinités locales, vénérées en qualité de protectrices du lieu concerné, dont il fallait capter les faveurs. L'évolution tendit à privilégier les divinités romaines chez les soldats qui avaient coutume de célébrer par un ex-voto une promotion ou un transfert ou n'importe quel événement heureux de leur carrière.

Summary: The article outlines the methodological difficulties raised by the interpretation of several private religious inscriptions from the western provinces of Rome, dedicated by soldiers or officers on duty, in the absence of a definite context. A particular attention is paid to the local gods, honoured as the numina of the place, whose protection was then needed. The evolution tended to favour the Roman deities among the military who were used to fulfil their vow to celebrate a promotion or a transfer or any happy event in their career. 
(Página deixada propositadamente em branco) 


\title{
SOLDATS ET CULTES INDIGÈNES DANS LES PROVINCES OCCIDENTALES AU HAUT-EMPIRE*
}

\author{
à la mémoire de John Cecil Mann
}

Les inscriptions religieuses d'époque romaine sont, avec les inscriptions funéraires, les miroirs plus ou moins déformants des préoccupations et des modes de raisonnement des soldats dans l'exercice ordinaire de leur métier au diapason du déroulement de leur carrière. L'examen de leur contenu sous cet angle est un des outils parmi les plus utiles pour qui cherche à écrire l'histoire quotidienne d'une armée provinciale, en l'absence d'autres sources directes d'information. En l'occurrence, le caractère militaire des documents ne signifie pas que les soldats étaient, en la matière, coupés des civils, ni qu'ils développaient ès qualités des croyances ou des traits de mentalité spécifiques. Simplement, le cadre dans lequel se déroulait leur existence influait de manière particulière sur l'expression religieuse de leurs attentes, de leurs espoirs et de leurs dévotions. L'armée romaine était, à n'en pas douter, un puissant instrument d'intégration et les sources indiquent que la religion officielle, celle qui déterminait les rites, les célébrations et les fêtes collectives, obéissait aux règles et aux évolutions de la religion civique ${ }^{1}$.

* Je remercie vivement mon ami J. d'Encarnação d'avoir accepté ce travail dans la revue Conimbriga. L'article, rédigé dès 1995 et prévu initialement pour la revue Sintria, a pu faire l'objet de quelques compléments et améliorations. Je remercie aussi C. Feuvrier-Prévotat et N. Moine qui m'ont accueilli chaleureusement et m'ont donné l'occasion d'exposer la question et d'en débattre lors d'un séminaire à l'Université de Reims le 8 décembre 2001.

1 Cf. A. von Domaszewski, Die Religion des römischen Heeres, Aufsätze zur römischen Heeresgeschichte, Darmstadt, 1972 (réimp.), p. 81-204; E. Birley, The religion 
Les dédicaces des autels des bénéficiaires d'Osterburken ou de Sirmium $^{2}$, les inscriptions religieuses de Novae ${ }^{3}$, le camp de la légion I Italica, illustrent au niveau local le caractère profondément romain de la religion militaire qu'il s'agisse du panthéon ou de la faculté à acclimater de nouvelles divinités ${ }^{4}$. À l'inverse, un sanctuaire de tradition indigène, comme celui du dieu Endovellicus en Lusitanie, n'a livré, à ce jour, qu'une dévotion possible - mais non certaine - d'origine militaire parmi plus de quatre-vingts ex-voto ${ }^{5}$. L'éloignement relatif des zones

of the Roman army (1895-1977), ANRW, II, 16, 1978, p. 1506-1541; A. D. Nock, The Roman army and the Roman religious year, Essays on religion and the Ancient world, Oxford, 1972, p. 736-790. Cf. aussi le bilan proposé par I. P. Haynes, The romanization of religion in the auxilia of the Roman imperial army from Augustus to Septimius Severus, Britannia, 24, 1993, p. 141-157 (il semble ignorer cependant l'article de G. Alföldy, infra, n. 61), qui, s'interrogeant sur la religion comme instrument d'intégration culturelle entre Auguste et Septime Sévère, situe bien le problème des liens religieux du soldat dans leur diversité. Récemment, G. L. Irby-Massie, Military religion in Roman Britain, Leyde-Boston-Cologne, 1999 (= Irby-Massie, Religion), a tenté d'analyser les rapports entre les soldats et les dieux dans le cadre d'une province où la part de l'épigraphie militaire est prépondérante. Elle suggère en particulier que la distinction entre les divinités locales et les divinités non locales n'était pas une préoccupation pour la majorité des individus, mais cette présentation tend à confondre les différents niveaux de l'expression religieuse et de la dévotion individuelle et collective.

2 E. Schallmayer et al., Der römische Weihebezirk von Osterburken I. Corpus der griechischen und lateinischen Beneficiarier-Inscriften des römischen Reiches (CBFIR), Stuttgart, 1990. M. Mirković, Beneficiarii consularis in Sirmium, Chiron, 24, 1994, p. 345-404. On dispose aussi, depuis peu, de la synthèse fondamentale de J. Nelis-Clément, Les beneficiarii: militaires et administrateurs au service de l'empire ( ${ }^{e r}$ s. a.C. $-V I^{e}$ s. p.C.), Bordeaux, 2000 (Ausonius-Publications. Etudes, 5) (= NelisClément, Beneficiarii).

3 V. Božilova, J. Kolendo, L Mrozewicz (sous la direction de J. Kolendo), Inscriptions latines de Novae, Poznan, 1992 (=ILNovae). En outre, pour les documents connus jusqu'en 1995, Inscriptions grecques et latines de Novae (Mésie inférieure) (= IGLNovae), J. Kolendo, V. Božilova éd., Bordeaux, 1997 (Ausonius-Publications. Mémoires, 1).

4 Voir la synthèse très suggestive de J. Nelis-Clément, Le monde des dieux chez les beneficiarii, Der römische Weihebezirk von Osterburken II. Kolloquium 1990 und paläobotanische-osteologische Untersuchungen, Stuttgart, 1994 (= Nelis-Clément, Monde des dieux), p. 251-260, qu'on complètera par Nelis-Clément, Beneficiarii, p. 133-210 en particulier. Ce dernier travail a pu, à la différence du précédent, tenir compte de la publication des autels de Sirmium (ci-dessus n. 2).

5 S. Miguel da Mota, Terena, Alandroal: J. d'Encarnação, Inscrições romanas do conventus pacensis $(=I R C P)$, Coimbra, 1984, n. ${ }^{\circ} 482$ à 565, p. 561-629. 
de garnison fournit une explication commode à cette faible proportion, mais on peut d'emblée constater que les divinités indigènes, c'est-àdire de nom local interprété ou non, ne représentent en général qu'une faible part des dédicaces d'origine militaire. Il convient donc de rechercher dans quelles conditions ou circonstances le dialogue se nouait avec les numina locaux. L'évolution même des cultes indigènes et des attitudes religieuses des soldats de métier devrait en être mieux éclairée.

\section{Questions de méthode.}

L'analyse de la documentation dans le but indiqué appelle des précautions particulières tant en ce qui concerne l'interprétation des textes que l'élaboration d'une méthode pour y parvenir. Deux exemples permettront de situer les problèmes et les écueils.

1. La dédicace provient de Novae, dans la province de Mésie inférieure. Le piédestal, trouvé dans la basilique épiscopale, avait été placé à l'origine dans les principia du camp légionnaire ${ }^{6}$ :

Pro salute I[mp(eratoris)] / Victoriae Augustae / Pantheae sanctis/ /simae L. Maximius / L.f. Voltiniae Gaetu/licus Viennae quod / tiro aput leg(ionem) XX V(aleriam) V(ictricem) / voveram nunc / $p$ (rimus) $p$ (ilus) leg(ionis) I Ital(icae) stip(endiorum) / LVII v(otum) s(olvi) / Marullo et Aeliano co(n)s(ulibus) $)^{7}$.

La promesse visait sans doute l'issue d'une carrière à la fois réussie et à l'abri des embûches du métier militaire. Le mérite n'était pas mince, pour un soldat sorti du rang (tiro), d'atteindre le centurionat, puis le primipilat. L'âge avancé et la longueur du service nécessaire à l'obtention du premier rang font écho à Juvénal et ne peuvent surpren$\mathrm{dre}^{8}$. Le parcours du centurion, citoyen de la colonie romaine de Vienne

6 ILNovae, $27=$ AE, 1985, 735.

7 Pour la sauvegarde de l'empereur, à la Victoire auguste et très sainte panthée, L. Maximius Gaetulicus, fils de Lucius, de la tribu Voltinia, originaire de Vienne; la promesse que jeune recrue de la XX légion Valeria Victrix j'avais contractée, maintenant primipile de la Ire légion Italica ayant accompli 57 années de service, je l'ai acquittée, sous le consulat de Marullus et d'Aelianus (= 184 p. C.).

8 Juvénal, Sat., XIV, v. 191-201: en particulier le ut locupletem aquilam tibi sexagesimus annus adferat. 
en Gaule Narbonnaise, commencé en Bretagne, dans la vingtième légion, sous Hadrien en 127 p. C., s'est achevé en Mésie inférieure sous Commode. Deux autres textes permettent de savoir que c'est aussi en Bretagne qu'il a obtenu le centurionat ${ }^{9}$, mais on ne peut pas dire à quelle date il est arrivé dans le secteur danubien, ni s'il a participé aux guerres de Marc Aurèle ${ }^{10}$. Recruté, d'après les statistiques, entre 17 et 20 ans, Gaetulicus était âgé de 74 à 77 ans et était peut-être sur le point de se retirer. Peu fréquente, la Victoire à la fois auguste et panthée réunissait les pouvoirs divins dans leur ensemble, ceux qui protégeaient l'armée comme ceux qui, de manière plus personnelle, avaient veillé sur le soldat tout au long de sa longue carrière. L'autel est un indice, parmi d'autres, que les soldats étaient coutumiers de vœux aux différentes étapes de leur parcours professionnel, depuis le moment où ils étaient recrutés après une période probatoire jusqu'à son terme - toujours incertain -, et rend compte d'un nombre relativement élevé d'ex-voto qui accompagnaient promotions et missions provinciales ${ }^{11}$.

2. L'inscription, provenant de Duratón, dans la province de Ségovie, est un piédestal en calcaire, encastré dans le mur d'une maison particulière. Elle a été réétudiée récemment par R. C. Knapp ${ }^{12}$ :

${ }^{9}$ RIB 1725 et 2120 ; ce sont des dévotions à Jupiter Dolichenus, pour l'une, et à Apollon, pour l'autre.

10 La dédicace à la Victoire est un indice qu'il a eu une vie vouée à la gloire de l'empereur, mais aussi marquée par les combats contre les barbares.

11 Cf. par ex. Schallmayer n. ${ }^{\circ}$, n. ${ }^{\circ} 259$, n. ${ }^{\circ} 918$ ou encore les autels des bénéficiaires de Sirmium: $n .^{\circ} 6,7$ et 8 où les vœux sont faits iterata statione ou parce que le bénéficiaire a la chance de servir in patria sua. On ajoutera $R I T, 33 \mathrm{a}=A E, 1998,803$ et 1999, 967: I(oui) O(ptimo) M(aximo et / Fortunae reduci et / Genio leg(ionis) VII G(eminae) P(iae) F(elicis) / Q. Cae(lius) Felix / aram / quam specul(ator) voverat / accepta / honesta missione / ex comment(ariensi) / praesidis / p(rovinciae) H(ispaniae) C(iterioris) / l(ibens) posuit. [Traduction: À Jupiter très Bon et très Grand et à la Fortune du bon retour et au Génie de la VII légion Gemina Pia Felix, Quintus Caelius Felix a fait placer de bon gré l'autel que speculator il avait promis, après avoir reçu son congé honorable comme commentariensis du gouverneur de la province d'Espagne citérieure.] Tous les autels et ex-voto ne précisaient pas, à toutes les époques, le détail des promesses, mais on ne peut douter que, dans une majorité de cas, il existait un lien avec les péripéties du métier.

12 LICS , n. ${ }^{\circ} 291$ avec dessin et photo. L'auteur parle d'un autel, ce qui n'est pas absolument sûr d'après l'apparence de la face inscrite présentant un cadre mouluré, élément compatible cependant avec une ara dans les contextes locaux de la péninsule occidentale, comme le prouvent plusieurs exemples. 
Fortunae / balneari / sac(rum) Vale/rius Buc/co miles / leg(ionis) II Adiultricis P(iae) F(idelis) / (centuria) Aemili S/ecundi / [- - -].

Le surnom du soldat, lu Tucco auparavant, a été corrigé en Bucco, qui est donné comme sûr, et le prénom $Q$. (= Quintus) est à lire comme une hedera. La Fortuna balnearis était la divinité tutélaire de l'établissement thermal, public plutôt que privé, où elle avait un sanctuaire susceptible de recevoir les vœux des usagers. Il n'y a pas, a priori, de critère satisfaisant de datation en dehors du terminus post quem fourni par la création de la légion: un intervalle entre l'époque de Vespasien et celle de Septime Sévère peut être retenu, sans plus de précision.

Ce miles est le seul de cette unité à être attesté en Hispanie. Toutefois, à Astorga, un vétéran de la légion II Adiutrix, originaire de Braga, est enterré par les soins de son fils, également enrôlé dans cette même légion ${ }^{13}$. Le texte date sans doute de la première moitié du $\mathrm{II}^{\mathrm{e}}$ siècle, mais la participation du fils à la dédicace du tombeau, aux côtés d'un affranchi, n'est pas la preuve que l'unité était alors en service dans la péninsule. La seule certitude est l'existence d'un recrutement hispanique à destination de la légion II Adiutrix qui se trouvait normalement en garnison à Aquincum depuis Trajan ${ }^{14}$. D'un strict point de vue onomastique, le cognomen Bucco du soldat de Duratón, à consonance celtique, n'est pas incompatible avec une origine hispanique et notamment celtibérique $^{15}$. Cependant, la mention de la centurie par le nom et le

13 Cf., en dernier lieu, F. Diego Santos, IRP León, 80, p. 96-97.

14 Cf. $R E$, XII, 149-1450: l'itinéraire suivi offre des aspects compliqués et conduit en Bretagne puis en Mésie; ce n'est que sous Trajan que l'unité s'installa à Aquincum, en Pannonie inférieure. Elle avait participé à la guerre dacique - tout comme la légion VII Gemina - au terme de laquelle un contingent de ses vétérans avait été établi dans la colonie Ulpienne de Poetovio: CIL, III, 4057.

15 C'est l'hypothèse privilégiée par P. Le Roux, L'armée romaine et l'organisation des provinces ibériques d'Auguste à l'invasion de 409, Paris, 1982 (= Le Roux, Armée), p. 159. Sur Bucco en Hispanie: J. M. Abascal Palazón, Los nombres personales en las inscripciones latinas de Hispania, p. 305. M. Sentius Bucco, centurion de la cohorte I Gallica, à Villalís, en 175 p. C. (AE, 1910, 1 = Le Roux, Armée, n. ${ }^{\circ} 246$, p. 243) est caractéristique de l'ambiguïté des conclusions fondées uniquement sur l'onomastique. R. C. Knapp tire argument du cognomen Bucco (et non plus Tucco) en faveur d'une origine cisalpine, possible en théorie, mais non certaine: la Bretagne, la Gaule narbonnaise offrent d'autres solutions acceptables. Cf. désormais $H E p, 4$, n. ${ }^{\circ} 228=A E, 1991,963$, relatif à un Cauriensis; ce qui montre bien qu'il s'agit d'onomastique locale, influencée par le substrat celtique (ce qui renvoie à un environnement 
surnom du centurion est un indice décisif que le soldat est en service actif au moment où il a élevé le monument à la Fortuna balnearis.

$\mathrm{Ne}$ faut-il pas alors envisager un séjour ne serait-ce que temporaire de l'unité en Hispanie? Duratón se trouvait près de la route entre Clunia et Ségovie, à l'écart il est vrai des axes de circulation donnés par les itinéraires ${ }^{16}$. Cette localisation n'est pas incompatible avec une mission spéciale (courrier, travaux d'utilité publique, ravitaillement ou fournitures, police etc.) qui avait alors pour destination des villes plus importantes, voire des capitales ou aurait eu trait à la construction d'une voie militaire. Un camp ou une origo paraissent être également des solutions possibles étant donné le dossier relatif aux liens connus à ce jour qui ont pu se nouer entre l'Hispanie et la légion II Adiutrix. Les motifs de l'affectation d'Hispani dans cette unité entre Vespasien et Trajan ne manquent pas et rien ne permet de dire que l'autel de Duratón n'était pas le résultat d'un ancien vœu que le soldat a acquitté par l'intermédiaire d'un proche et en son absence ${ }^{17}$.

Les exemples étudiés reflètent les difficultés auxquelles s'expose toute interprétation; ils concernent des inscriptions religieuses qui ne peuvent pas être traitées de la même manière que les épitaphes. Celles-ci, en l'absence d'un contexte familial bien identifié, sont de bons indices de la présence d'une structure militaire durable, voire permanente, à proximité, qu'il s'agisse d'un camp auxiliaire ou légionnaire, du séjour d'une vexillation ou de personnels au service d'un magistrat

culturel et non à l'origine). Au fond, la nouvelle lecture ne modifie pas vraiment les données essentielles.

16 Cf. J. A. Abásolo, Las vías romanas de Clunia, Burgos, 1978, p. 48-49. Les efforts de R.C. Knapp pour situer Nova Augusta à Duratón à l'aide de Ptolémée, p. 262-267, montrent que la solution ne peut pas être rejetée; elle se heurte surtout à un manque d'information incontestable (son absence des itinéraires et les difficultés propres aux localisations à partir de Ptolémée seul) pour une région de la péninsule où on constate que les toponymes antiques ont davantage été remplacés au cours des âges que dans d'autres secteurs géographiques. Toutefois, malgré H. Gimeno et M. Mayer, Una propuesta de identificación epigráfica: Lara de los Infantes/Nova Augusta, Chiron, 23, 1993, p. 313-321, le texte de Pline l'A., qui place la ville entre Ségovie et Termes (selon des itinéraires, puisqu'il ne suit pas ici l'ordre alphabétique, ni le critère des statuts), oriente vers la zone de Duratón.

17 La divinité invoquée, protectrice de la santé, constitue peut-être un indice sur les circonstances particulières de la dédicace. 
ou du gouverneur provincial, y compris une station de bénéficiaire ${ }^{18}$. Cependant, l'intervention de la famille n'est pas exempte d'ambiguïtés; elle ne signifie pas toujours que les restes du soldat défunt ont été déposés dans une tombe familiale, comme elle ne prouve pas que l'épouse et les enfants avaient accompagné le sous-officier dans sa résidence administrative. On le voit, seul un faisceau de présomptions, intégrant aussi l'histoire du recrutement provincial et la chronologie comme références utiles, est susceptible d'étayer convenablement un résultat. Le critère onomastique est en lui-même très délicat à manier, car l'expansion des noms romains ou romanisés a abouti, dans de nombreux cas, à les déraciner, c'est-à-dire à les rendre moins caractéristiques d'une origine précise.

C'est pourtant une des questions délicates et fondamentales à laquelle la recherche est confrontée. Une inscription militaire trouvée hors du contexte d'une garnison identifiée traduit-elle un lien personnel et familial du soldat avec la région de provenance du document ou bien n'est-elle que la trace d'un déplacement temporaire dans le cadre du service, étant entendu que le système des permissions existait? ${ }^{19}$ Une inscription votive, dans l'ignorance de la présence plus ou moins proche d'un camp, suscite des points d'interrogation semblables à ceux qu'appellent certaines inscriptions funéraires quand il s'agit de déterminer les motifs de l'élévation d'un ex-voto en un lieu donné. C'est le cas de la dédicace d'un signifer de la Ire cohorte Gallica trouvée à Rairiz da Veiga, Ginzo de Limia, dans la province d'Orense ${ }^{20}$ :

3. V(otum) s(olvit) l(ibens) m(erito) / Bandu(a)e / Veigebr/eaego / M. Siloni/us Gal(eria) Sillanus / sig(nifer) coh(ortis) I / Gall(icae) c(ivium) R(omanorum).

Le texte est datable du dernier tiers du $\mathrm{I}^{\mathrm{er}}$ siècle ou du début du $\mathrm{II}^{\mathrm{e}}$. La divinité invoquée est une divinité topique dont l'épithète se retrouve

18 Celles-ci se signalent toutefois surtout par des ex-voto en très grande majorité (67\% des autels votifs émanant de ces sous-officiers attestent une station). On ajoutera que, selon Nelis-Clément, Monde des dieux, p. 259, 13,3\% des bénéficiaires qui ont été affectés à une statio ont servi à proximité d'un camp ou d'un fortin. Voir également Nelis-Clément, Beneficiarii, p. 38-42.

19 R. W. Davies, The daily life of the Roman soldier, $A N R W$, II, 1, 1974, p. 333.

20 Le Roux, Armée, n. ${ }^{\circ}$ 162, p. 217, avec les références. 
par pur hasard dans le nom de la localité moderne (Veiga). L'indication de sa tribu Galeria de la part du signifer est notable dans la mesure où c'est peu habituel dans une dédicace religieuse ${ }^{21}$. Le lieu de garnison de la cohorte est ignoré, mais on a proposé récemment de la localiser à Porto Quintela, Baños de Bande (toponyme dérivé de Bandua), où on situe aussi Aquae Querquennae. Rairiz et le site de ce qui serait le camp se trouvent de part et d'autre des monts de Bande: à vol d'oiseau, la distance entre les deux est d'une vingtaine de kilomètres. Mais une mansio ou établissement routier n'est pas à exclure dans le cas d'Aquae Quer$q_{\text {uennae }} 22$. En outre, aucune autre inscription militaire n'est attestée dans le secteur et on peut faire état à Castrelo do Val, près de Verín, dans la province d'Orense, d'une dédicace émanant d'un sous-officier d'origine locale servant dans une unité qui ne se trouvait pas obligatoirement dans la région, ni même dans la péninsule ${ }^{23}$. On ajoutera que c'est dans le camp que le signifer de la I ${ }^{\text {re }}$ cohorte Celtiberorum s'est acquitté de son vœu ${ }^{24}$ et que les castra de la I $^{\text {re }}$ cohorte Gallica sont situés à Veleia (Iruña, Alava) par la Notice des Dignités, sans qu'il soit question de modification récente comme dans le cas de la cohorte des Celtibères ${ }^{25}$. La tessère de Castromao, trouvée à une trentaine de kilomètres d'Aquae Querquennae a pu faire penser que le "camp" était celui de cette unité, mais il a été localisé ailleurs depuis, comme nous venons de le voir. Dans la mesure où la dédicace est strictement indivi-

21 Ce n'est pas sans exemple et la tribu Galeria peut indiquer une citoyenneté relativement ancienne; elle est présente à Lugo, semble-t-il. Sur les tribus et leur développement dans la péninsule, voir la synthèse d'A. U. Stylow, Veleia, 12, 1995, p. 105$-123=A E, 1995,705$.

22 Cf. P. Le Roux, L'armée romaine dans la péninsule Ibérique sous l'Empire: bilan pour une décennie, REA, 1992, p. 234 (= Le Roux, L'armée). En tout état de cause, un camp n'est pas davantage exclu, mais la chronologie serait alors peut-être à affiner, voire à réviser. En outre, comme on verra infra, si on devait retenir un camp, il s'agirait de celui de la cohorte Gallica.

23 Il s'agit de la dédicace à I.O.M. par L. Cas. Caen.: Le Roux, Armée, p. 88.

24 Aujourd'hui bien localisé à Sobrado dos Monxes, La Corogne: $C I R G, \mathrm{I}, \mathrm{n}{ }^{\circ} 32$. P. Le Roux, L'armée, p. 233 et p. 253 , n. ${ }^{\circ} 21$.

25 Not. Dig., Occ., XLII, 25-32. Il est toutefois peu vraisemblable, au regard de la documentation épigraphique locale, que le camp de la cohorte ait été durant le Haut-Empire localisé près de cette agglomération. C'est par sa participation à la surveillance des sites miniers, sous forme de vexillations, que la cohorte est attestée par ailleurs, ce qui n'indique rien sur l'emplacement du camp. 
duelle, on ne peut pas exclure une mission du signifer ${ }^{26}$. C'est aussi ou dans le cadre de l'exercice ou dans le camp lui-même qu'il a pu s'acquitter de son vœu, à moins qu'il n'ait fait placer le monument dans un sanctuaire de son lieu d'origine, vraisemblablement à l'occasion de sa promotion comme porte-enseigne ou pour obtenir le succès dans l'exercice de ses nouvelles fonctions. Puisque la mention de la tribu Galeria n'est pas accompagnée de l'origo et s'ajoute à une onomastique de type celtique, on ne peut pas dire si le soldat est originaire du lieu ou de la région où a été trouvée l'inscription ou s'il est venu d'ailleurs. On ne peut en aucun cas exclure que le camp de l'unité ait été situé dans le voisinage de Rairiz da Veiga, alors que le site de Porto Quintela n'est pas le seul emplacement indiqué, ni complètement assuré ${ }^{27}$.

La dévotion à Bandua Veigebreaegus possède également la particularité de nous mettre en présence d'une invocation à une divinité indigène de la part non seulement d'un gradé d'unité auxiliaire, mais encore d'un citoyen romain. Le sens le plus immédiat que l'on prête à l'acte du signifer est alors celui d'obtenir une protection renouvelée de la part du dieu qui a favorisé la promotion ou qui représente le pouvoir divin dans la région où l'activité du soldat dans son nouveau grade doit se dérouler ou est en train de se dérouler. Loin d'apporter une

26 Le signifer n'était pas en principe en charge du détachement quand il participait à une vexillation. Il avait pour fonction d'ouvrir la marche lors de déplacements ou au combat; il était évidemment responsable des enseignes et de leur chapelle où étaient en outre placés les dépôts en argent des soldats: cf. par ex., Y. Le Bohec, L'armée romaine, Paris, 1989, p. 51, 53 et 233. On pourrait alors envisager une surveillance de la route ou de bâtiments publics, sans écarter un déplacement individuel dans l'intérêt du service et de nature indéterminée.

27 On ne peut pas, en tout état de cause, se fonder sur ce texte à vocation religieuse pour établir la présence d'un camp à Aquae Querquennae. Pour un bilan visant à asseoir définitivement l'hypothèse à la fois d'un fort auxiliaire et d'une mansio routière: A. Rodríguez Colmenero, S. Ferrer Sierra, F. M. Herves Raigoso, El complejo arqueológico romano de «Aquis Querquennis», Porto Quintela (Ourense), Los origenes de la ciudad en el Noroeste hispánico, Actas del Congreso internacional Lugo 15-18 de Mayo 1996, A. Rodríguez Colmenero coord., p. 891-910. Le camp, utilisé pour les travaux routiers, aurait précédé la mansio qui aurait subsisté seule dès l'époque de Trajan. La méthodologie appliquée appelle cependant quelque réserves avant qu'on puisse accepter pleinement ces conclusions. En tout état de cause, le signifer n'indique rien ni dans un sens ni dans un autre: ce sont les beneficiarii et non les signiferi qui étaient chargés de la surveillance de la circulation routière. L'hypothèse de la participation à des travaux routiers de la part d'auxiliaires seuls me paraît improbable. 
solution tranchée à la question de la garnison et de l'origo, la dimension religieuse de l'inscription maintient l'ambiguïté: il peut s'agir de capter la bienveillance des pouvoirs divins locaux parce qu'on est confronté momentanément à leur présence ou parce qu'au contraire ils président à la protection des lieux où est installée durablement la garnison.

\section{Un corpus limité.}

Le dossier hispanique en la matière n'est pas très fourni. On ne compte à ce jour que six dédicaces entrant sûrement dans la catégorie des dévotions à des divinités indigènes de la part de militaires ${ }^{28}$. Classées dans l'ordre chronologique, elles sont numérotées à la suite des témoignages déjà analysés:

4. Santo Tirso, Espagne citérieure (fig. 1).

CIL, II, 2374=5551.

L. Valerius Silvanus / miles leg(ionis) VI Vict(ricis) / [de]o Turiaco / v(otum) s(olvit) l(ibens) m(erito).

Le document est assurément à mettre en relation avec le séjour de la VI Victrix en Hispanie entre Auguste et l'avènement de Galba ${ }^{29}$. La période postérieure à la censure de Claude est probable puisque le soldat porte un cognomen. Rien n'indique son origine exacte et c'est dans le cadre d'une mission que la dédicace a été effectuée, car le camp de la VI Victrix se trouvait dans la région d'Astorga-León plutôt que dans le secteur de Braga. La divinité Turiacus n'est pas attestée par ailleurs; son caractère topique est vraisemblable.

5. Numão, Lusitanie (fig. 2).

CIL, II, 432; Fouilles de Conimbriga II, n. ${ }^{\circ} 3$, p. 21.

Ti. Claudius / Salicius ${ }^{30}$ eq(ues) / chor(tis) III Lusitanorum / dis

28 Sous réserve de documents inédits ou publiés confidentiellement, voire de documents qui pourraient entrer indirectement dans cette série parce que trouvés dans l'environnement d'un camp. Le faible nombre des occurrences ne permet aucune conclusion ferme, mais cette donnée peut signifier que les vœux adressés à des divinités indigènes répondaient à des conditions particulières tenant à la qualité du dévot ou à des aspects religieux malheureusement non précisés et décisifs aux yeux du dédicant.

29 Cf. P. Le Roux, Armée, p. 120, 182-183 et 353.

30 J. d'Encarnação, Divindades indígenas sob o domínio romano em Portugal. 
deabusq(ue) coniumbric(ensibus ou ensium) / [v(otum)] s(olvit) l(ibens) m(erito).

La citoyenneté remonte à Claude ou Néron. Le cavalier, originaire de Lusitanie, probablement de la civitas de Conimbriga, sert dans une cohorte de Lusitaniens dont la formation pourrait être récente ${ }^{31}$. Rien n'indique que l'unité est en garnison en Lusitanie et le caractère frontalier du lieu de découverte, associé au fait que le soldat s'en remet aux divinités de Conimbriga, milite en faveur d'un vœu à l'occasion d'un retour au pays plutôt que d'un départ pour le service ${ }^{32}$. La cohorte stationnée en Germanie ne convient pas, dans la mesure où elle n'est pas attestée comme cohorte montée ${ }^{33}$. Faut-il admettre qu'il s'agit d'une cohorte basée en Hispanie citérieure, mais non retrouvée par ailleurs dans la documentation hispanique? On comprendrait mieux l'emplacement de la dédicace et la vraisemblance irait alors vers une cohorte montée, créée sous Claude et recrutant encore dans le territoire d'origine. Les dii deaeque conimbricenses, divinités protectrices de la communauté et de ses habitants, englobent divinités indigènes et divinités romaines installées officiellement dans la cité pérégrine. Il est notable que le soldat les associe dans une même dévotion alors qu'il est devenu citoyen romain.

6. Territoire d'Igaedis (Idanha-a-Velha), Lusitanie (fig. 3). EE, VIII, $15=I L S, 4510$.

Ara(m) pos(uit) / Toncius / Toncetami / f(ilius) Icaedit(anus) / milis / Trebarune / l(ibens) m(erito) v(otum) s(olvit).

Subsídios para o seu estudo, Lisbonne, 1975 (= Divindades indígenas), p. 176, corrigeait déjà, à partir d'une ancienne photographie communiquée par Pinto Ferreira, le Sancius du CIL en Sailcius. R. Étienne et G. Fabre, Fouilles de Conimbriga, n. ${ }^{\circ}$ 3, p. 21, suggèrent Sanecius, mais donnent une photographie qui révèle que l'inscription a été détériorée depuis la lecture du CIL. Au regard des indices onomastiques et des critiques formulées à l'encontre de telle ou telle solution, la photographie invite à adopter Salicius, attesté (NPILH, p. 494).

31 On ne connaît avec certitude actuellement qu'une seule III ${ }^{\mathrm{e}}$ cohorte Lusitanorum, stationnée en Germanie inférieure jusqu'à l'époque de Vespasien et cantonnée en Pannonie à partir de Trajan: G. Alföldy, Die Hilfstruppen der römischen Provinz Germania inferior, ES, 6, 1968, p. 65-66 et 208-209 en particulier.

32 L'interprétation est délicate, car le cavalier ne dit pas qu'il est vétéran, et la citoyenneté a pu être acquise en cours de service aussi bien qu'à la fin.

33 Cf. la mise au point de N. Santos Yanguas, Las cohortes Lusitanorum en el ejército romano, Conimbriga, 18, 1979, p. 188-192. 
Le même soldat, originaire de la cité d'Igaedis, a pris soin de faire élever une dédicace à la Victoire alors qu'il était devenu signifer de la $\mathrm{II}^{\mathrm{e}}$ cohorte des Lusitaniens ${ }^{34}$. De statut pérégrin, il s'en est remis à une divinité locale pour le succès d'une carrière qui a commencé à l'époque julio-claudienne. Trebaruna, mal connue, mais attestée par ailleurs, apparaît comme liée à la Lusitanie centrale et aux différents populi désignés sous le nom de Lusitaniens ${ }^{35}$.

\section{Caleruela, Tolède.}

$A E, 1988,823$ et Le Roux, L'armée, n. ${ }^{\circ}$ 14, p. 251.

Bassus / Turobei (filius) / eques al/ae Vetto/num ara/m posit (sic) / Ataecin/ae votu/m solv/it m(erito) / - - -

Comme l'a montré G. Alföldy ${ }^{36}$, le cavalier a servi selon toute vraisemblance dans l'ala Hispanorum Vettonum civium Romanorum qui se trouvait en Bretagne entre l'époque flavienne et la fin du $\mathrm{II}^{\mathrm{e}}$ siècle, mais peut-être aussi dès l'époque claudienne. L'absence de civium Romanorum militerait en faveur d'une date préflavienne. L'inscription pourrait même être le témoin d'un séjour originel de l'unité dans la péninsule ${ }^{37}$. Comme pour l'inscription précédente la formule aram posuit est utilisée en plus du votum solvit. Ataecina se rencontre à plusieurs reprises dans divers lieux de la Lusitanie et de l'Espagne citérieure, accompagnée en particulier de l'épithète Turobrigensis, qui rappelle le nom Turobeius du père du cavalier ${ }^{38}$. Divinité topique et tutélaire, Ataecina semble appartenir prioritairement à la région d'Estrémadure

34 ILS, 4510 a. Une cohorte de même nom serait mentionnée dans une inscription d'Égypte (CIL, III, 13582) tandis qu'à Salone en Dalmatie est recensé un préfet d'une cohorte de même nom et même numéro (CIL, III, 8733). On ne possède pas d'éléments suffisants pour identifier la cohorte mentionnée à Idanha avec l'une ou l'autre et encore moins pour lui attribuer une garnison qui ne saurait être située, sans plus d'indices, dans la province de Lusitanie.

35 Cf. par ex. J. d'Encarnação, Divindades indígenas, p. 288-291.

36 G. Alföldy, Römische Heeresgeschichte, Amsterdam, 1987, p. 517-519.

37 Toutefois, comme le note G. Alföldy lui-même, le territoire d'où provient le document était englobé dans celui des Vettons. Dans la mesure où, sous l'Empire, les unités ne sont pas, le plus souvent, installées dans la région où elles ont été recrutées, il est probable que le soldat exprime son attachement à sa cité d'origine plus qu'il ne révèle le séjour de l'unité sur le territoire.

38 J. M. Blázquez Martínez, Religiones primitivas de Hispania, Madrid, 1962, p. 147. Voir aussi $A E, 1995,734-760$. 
autour de Mérida et Cáceres. Plutôt qu'à un camp ou à une mission dans la région de garnison, le texte fait penser à la dévotion d'une nouvelle recrue se plaçant sous son patronage en vue de son service.

8. Cornoces, Orense, Espagne citérieure.

IRG, IV, 92 = Le Roux, L'armée, n. ${ }^{\circ}$ 87, p. 196.

Moelio / Mordo/niego / L. Caecili/us Fusc/us m(iles) le(gionis) $V<I I>$ ? / G(eminae) F(elicis) v(otum) s(olvit) l(ibens) m(erito) // (à dr.) [I]III k(alendas) iun(ias) / Imp(eratore) Vespasiano / VIIII Tito VI co(n)s(ulibus).

Datée du 29 mai 79 p. C., la dédicace concerne un soldat détaché pour service dans la région. A. Tranoy pense qu'un lien est possible, au vu de la localisation du document, avec les travaux du pont de Chaves $^{39}$. Moelius Mordoniegus n'est pas connu par d'autres inscriptions, mais son nom s'apparente à celui de nombreuses divinités topiques ou tutélaires.

3. Cf. ci-dessus, n. ${ }^{\circ}$ 3, p. 107.

Dédicace du signifer de la $\mathrm{I}^{\mathrm{re}}$ cohorte Gallica à Bandua Veigebreaegus. La date est au plus tôt flavienne, selon toute vraisemblance.

On constate aisément, sur un échantillon il est vrai modeste, qu'il n'y a guère de témoignage postérieur au $\mathrm{I}^{\mathrm{er}}$ siècle et que les auxiliaires, souvent d'origine lusitanienne, adressent plus volontiers un ex-voto aux divinités indigènes que les légionnaires. Quel que soit le cas de figure, il s'agit de vœux à titre personnel dans le cadre de la carrière ou de l'activité militaire. Les citoyens romains sont au nombre de quatre et les pérégrins au nombre de deux. Toutefois, il est très probable que la totalité des dédicants était d'extraction provinciale et indigène. Le recours à des divinités locales s'explique ou par le souci de ne pas s'attirer les rancœurs des divinités protectrices du lieu ou par l'attachement à une divinité ancestrale.

Ce dernier trait semble s'estomper au fur et à mesure de l'évolution décelable dans la documentation des autres provinces de la pars Occidentis. Il y a quelques cas de référence à des cultes indigènes liés à l'origine des dédicants, comme à Birrens, un des forts du mur d'Ha-

39 A. Tranoy, La Galice romaine. Recherches sur le nord-ouest de la péninsule Ibérique dans l'Antiquité, Paris, 1981, p. 276. 
drien et de son système de surveillance ${ }^{40}$. Des soldats de la II $^{\mathrm{e}}$ cohorte des Tongres, originaires d'un pagus Condrustis (aujourd'hui Condrost dans la région de Liège), y invoquent la déesse Viradecthis protectrice de la cité des Tongres, tandis que ceux d'un pagus Vellaus, non localisé, honorent Ricagambeda, certainement aussi associée au territoire d'origine des dédicants ${ }^{41}$. Ce sont ensuite les divinités du lieu où est élevé l'ex-voto qui sont surtout remerciées, soit seules soit, le plus souvent, en association avec d'autres divinités du panthéon romain. Même s'il s'agit d'un officier, le cas de la dévotion à Covventina par le préfet de

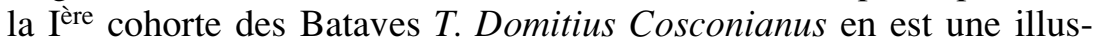
tration $^{42}$. On peut rappeler aussi l'inscription au deus Cocidius, désigné par ailleurs sous la forme de Mars Cocidius ou même de Silvanus Cocidius $^{43}$, offerte par un détachement de soldats de la $\mathrm{XX}^{\mathrm{e}}$ légion Valeria Victrix, alors qu'ils participaient à des travaux dans le secteur du mur d'Hadrien ${ }^{44}$. Mais ce sont les inscriptions de bénéficiaires qui traduisent le mieux la combinaison des numina ${ }^{45}$, comme elles soulignent la part prépondérante du panthéon romain traditionnel ${ }^{46}$.

Leur originalité tient surtout à la mise en valeur des circonstances dans lesquelles s'effectuaient les vœux et à la dimension à la fois privée et publique ou officielle de l'initiative religieuse du soldat. Le recensement des occurrences a montré, en effet, que l'arrivée dans une statio, une prolongation du poste, une promotion ou le départ pour une autre destination ou fonction constituaient les motifs les plus communs des

40 D. J. Breeze et B. Dobson, Roman officers and frontiers, Stuttgart (Mavors X), 1993, p. 465, p. 482 et p. 495-500: après une première occupation entre 80 et 87 p. C., il est bâti vers 128 p. C., puis reconstruit vers 158 p. C. L'abandon est à placer au début des années 180. C'est à partir de 157/158 que la cohorte II des Tongres est stationnée au fort de Birrens.

41 CIL, VII, 1073 = ILS, 4756 (aussi 4757-4759); CIL, VII, $1072=I L S, 4752$.

$42 R I B, 1534$. Il s'agit d'une divinité d'une source qui avait un sanctuaire près de Brocolitia depuis l'aménagement du mur d'Hadrien.

43 RIB, 2024 (près de Stanwix, Carlisle); CIL, VII, 642 (Housesteads) = ILS, 4723.

44 CIL, VII, 802 = ILS, 4722. La date consulaire Apro et Rufo cos n'est malheureusement d'aucun secours pour la datation du texte. Il serait essentiel de savoir où exactement l'inscription a été découverte par rapport au camp ou fort.

45 Cf. Nelis-Clément, Monde des dieux, p. 258-259 principalement.

46 Voir Nelis-Clément, Beneficiarii, p. 33-42 et 193-202, qui montre bien la dualité complémentaire des dédicaces, à la fois officielles et personnelles. 
actes religieux de ces militaires ${ }^{47}$. En outre, si un certain nombre de bénéficiaires associe des membres de la famille ou des collègues ${ }^{48}$, Jupiter et particulièrement Jupiter Optimus Maximus sont en majorité présents, ce qui indique une reconnaissance envers l'État romain et l'armée ${ }^{49}$. L'adjonction assez fréquente de la date consulaire révèle aussi la volonté de situer le vœu dans une durée qui est à la fois un temps personnel (anniversaire, fête à la quelle on attache personnellement de l'importance, valeur symbolique) et un temps inscrit dans la continuité du service et de la carrière. À Sirmium, il est notable qu'un bénéficiaire éprouve le besoin de préciser à la date de 205 p. C. Genio huius loci patriae suae ${ }^{50}$ : sans préjuger ici de la signification de l'invocation au Genius loci, la formule indique le souhait de ne pas servir trop loin des terres ancestrales et familières et renforce l'idée que tous ces vœux militaires expriment un point de vue sur le service et sur la possibilité d'éviter ses plus gros inconvénients. En revanche, la série de Sirmium ignore totalement les divinités indigènes, mais la cité était une colonie flavienne ${ }^{51}$ et les autels sont datés entre 157 et 231 p. C.

Une recherche non exhaustive, mais relativement étendue, dans les secteurs de Bretagne, des Germanies et des provinces danubiennes confirme, encore une fois, que les dévotions envers des divinités locales sont partout assez limitées. Toutefois, il n'y a pas d'exclusive depuis le simple soldat auxiliaire ou légionnaire jusqu'au centurion, au tribun ou au préfet. Les circonstances, autant que la chronologie, rendent compte de ces observations. Au-delà d'une simple idée de tolérance, quels sont les facteurs religieux qui entraient en ligne de compte et sous quel éclairage peut-on essayer de mieux les comprendre?

47 Nelis-Clément, Monde des dieux, p. 253. Les 79 documents et fragments du catalogue de Sirmium (Pannonie inférieure) confirment le diagnostic (cf. supra n. 2): par ex $\mathrm{n} .^{\circ} 1$ et $\mathrm{n} .^{\circ} 59$, missus honesta missione; $\mathrm{n} .^{\circ} 6$, iterata statione; $\mathrm{n} .^{\circ} 13$, iterata statione in patria sua; $\mathrm{n}{ }^{\circ} 8$, pro salute Augustorum, agens in statione.

48 Nelis-Clément, Monde des dieux, p. 255-256. Cependant, l'évolution va dans le sens d'un renforcement de la vie familiale au cours du $\mathrm{II}^{\mathrm{e}}$ et du $\mathrm{III}^{\mathrm{e}}$ siècle, alors qu'au $\mathrm{I}^{\text {er }}$ siècle c'étaient plutôt les commilitones qui tenaient lieu de famille.

49 Nelis-Clément, Monde des dieux, p. 252.

50 N. ${ }^{\circ} 11$ du catalogue de M. Mirković.

51 B. Galsterer-Kröll, Untersuchungen zu den Beinamen der Städte des Imperium Romanum, ES, 9, 1972, p. 125. 


\section{Une question de lieu?}

Dans la série même des dévotions militaires à des divinités indigènes, quelques distinctions sont nécessaires. On exclura du nombre le Genius loci surtout vénéré, comme on vient de le voir, par les bénéficiaires, en association avec d'autres divinités. En effet, rien ne permet de dire qu'il ne s'agit pas alors de la forme romaine du Genius susceptible de s'appliquer à un individu, à une entité ou communauté, à un bâtiment public ou à un lieu. Quand ce n'est pas le cas, la divinité topique d'origine indigène conserve son identité et ses attributs particuliers comme le montre l'exemple de Bandua Veigebreaegus. Cependant, la relation est relativement complexe et résiste souvent à l'analyse approfondie faute d'une connaissance satisfaisante du lieu de découverte dans ou aux alentours du fort. Lorsqu'un lien apparaît avec un établissement permanent, il n'y a jusqu'à présent pas d'exemple assuré que la divinité locale avait sa place parmi les dieux militaires présents à l'intérieur même du campement.

Dans un certain nombre de documents, la divinité indigène est seule et nommée de façon traditionnelle: outre Trebaruna ou Ataecina déjà étudiées, on peut évoquer les Matres Aufaniae et leur sanctuaire de Nettersheim en Germanie inférieure ${ }^{52}$ ou la dea Nehalennia honorée sur l'ancienne île de Noord-Beveland (province de Zélande) ${ }^{53}$. Dans ce cas, la divinité ne joue pas seulement le rôle d'une Tutela loci, expression utilisée par l'un des bénéficiaires qui s'adresse aussi aux deae Aufaniae, loin du sanctuaire de Nittersheim il est vrai, ce qui maintient l'ambiguiité ${ }^{4}$. Tantôt appelées matres voire matronae, tantôt désignées par le seul qualificatif d'Aufaniae, tantôt données comme deae Aufaniae, les divinités germaniques prennent un aspect variable selon le lieu et le dédicant, mais la protection qu'elles sont supposées accorder ne vise pas nécessairement l'intégrité physique plutôt que le succès d'une entreprise ou l'obtention d'une affectation particulièrement désirée.

52 Par ex. CBFIR, n. ${ }^{\circ}$ 51-53, 76-79.

53 CBFIR, n. ${ }^{\circ} 94$. Voir aussi P. Stuart et J. E. Bogaers, Römische Steindenkmäler aus der Oosterschelde bei Colijnsplaat, I, Textband, Leyde, 2001, particulièrement p. 20-25, sur les images.

54 CBFIR, n. ${ }^{\circ} 120$ (Mayence). Le dédicant, L. Maiorius Cogitatus était peut-être originaire de Germanie inférieure. 
C'est à l'ensemble des pouvoirs divins qui sont censés émaner des déesses qu'il est fait recours ou qu'il est adressé remerciement.

Les divinités dites ordinairement interprétées posent d'autres problèmes. Il s'agit, par exemple, d'Apollo Toutiorix qui a exaucé les prières du centurion L. Marinius Marinianus ${ }^{55}$; de Mars Cocidius vénéré par des soldats de la $\mathrm{II}^{\mathrm{e}}$ légion Augusta ${ }^{56}$; de Mars Halamardus qui a comblé le centurion T. Domitius Vindex de la XXe légion Valeria Victrix $^{57}$. Il semble que, dans tous ces cas, le nom de la divinité ne soit pas à interpréter seulement ou obligatoirement comme le résultat d'une assimilation de la divinité indigène par la divinité romaine. Comme dans le cas d'un Apollon ou d'un Mars auguste, qu'il faut comprendre avant tout comme l'Apollon ou le Mars qui protège et rend efficace le pouvoir impérial, le numen indigène conserve son efficacité par l'intermédiaire de la divinité romaine qui, c'est vrai, paraît la mieux désignée pour y parvenir. Le choix d'Apollon met l'accent sur le salut et la sauvegarde, celui de Mars sur le métier militaire et la protection de la communauté. Toutefois, il est remarquable que Cocidius soit aussi rendu bienfaisant par l'intervention de Silvanus ${ }^{58}$, ce qui révèle qu'il s'agit moins d'assimilation que de recherche de la meilleure formule divine à un moment donné. L'association hiérachisée des dieux n'avait pas pour critère fondamental le caractère romain et le caractère indigène, mais la dimension locale et la dimension politique pour des militaires souvent issus des sociétés provinciales et en voie d'intégration, voire complètement intégrés.

À vrai dire, l'attitude ainsi observée s'applique à toutes les régions et à tous les types de culte. Même s'il n'est pas directement lié à un soldat, le document de Novae relatif au deus Aeternus est à mettre en relation avec la vie du camp de la I ${ }^{\mathrm{re}}$ légion Italique ${ }^{59}$. L'actor Aurelius Statianus ${ }^{60}$, sauvé d'un grave danger en mer, acquitte son vœu en-

55 ILS, 4641, de Wiesbaden (Aquae Mattiacorum).

56 ILS, 4724.

57 ILS, 4561, de Roermond/Horn, lieu dit du Limbourg (Pays-Bas), proche de la Meuse.

58 ILS, 4723 (deus Silvanus Cocidius).

59 ILNovae, n. ${ }^{\circ}$ 4: le motif affirmé est periculo maris liberatus. On pense à une navigation sur la mer Noire.

60 Il pourrait s'agir d'un actor du primipile, comme on en connaît dans l'épigraphie: cf. P. Le Roux, L'armée, p. 235-236, mais on peut objecter que cela aurait été explicitement dit. Le fait qu'il soit libre, sans qu'on sache s'il était affranchi, implique 
vers une divinité qui est étroitement associée au territoire de la province voisine de Dacie et n'est guère attestée par ailleurs. Quelles que soient les origines et les qualités du dieu, le dédicant s'est placé, dans l'adversité, sous la protection d'une divinité qui lui était proche parce que familière et lui a donné droit de cité en un lieu où ses fonctions l'avaient appelé, en accomplissement de sa promesse (ex voto promisso). Mais ce document souligne une difficulté particulière de l'interprétation, car la provenance en est probablement l'extérieur du camp, ce qui n'a pas la même signification que s'il s'agissait de l'intérieur; on n'est malheureusement pas toujours en mesure de le déterminer.

Les différents éléments mis en évidence ne laissent pas de doute sur les relations qu'entretenaient les militaires avec les divinités locales dans le cadre de l'exercice de leur métier, mais l'explication du fait que ces dévotions aient été relativement minoritaires n'est pas immédiate ni univoque. D'un point de vue strictement religieux, c'est l'indice que loin d'exclure les dieux dits indigènes, la religion des soldats, à l'image de la religion civique, s'en accommodait et leur accordait une efficacité comparable à celle des autres divinités. En tout cas, leur vitalité exprimait leur intégration à l'édifice impérial pour le bien et la protection de tous les sujets. Associée de près au pouvoir impérial qu'elle avait aussi pour mission de représenter, l'armée contribuait à manifester la puissance de Rome autour de laquelle étaient rassemblés tous les numina vénérés dans l'Empire. Ceux-ci vivaient en quelque sorte par la volonté de l'empereur et assuraient en retour la bienveillance des puissances divines envers les tâches auxquelles Rome se sentait vouée.

Cependant, dans les milieux militaires comme dans le reste de la société, l'évolution juridique et politique marquée par la diffusion des cités et de la citoyenneté romaine a joué en faveur des dieux patrons et protecteurs de Rome, même si, avec les Sévères l'accent paraît avoir été mis sur l'universalité impériale et sur le rassemblement de toutes les énergies humaines et divines au service de l'État impérial. Une hiérarchie s'était établie au profit des dieux traditionnels de Rome et de l'Italie et c'est peut-être par le biais du culte impérial que s'exprimait le mieux le souci de convoquer tous les numina - petits et grands - au

la possibilité d'autres solutions. Le caractère public ou privé de la fonction n'est pas davantage décelable. La diffusion restreinte du culte milite en faveur d'une origine dace de Statianus, mais là encore on doit ménager le recours à d'autres hypothèses. 
service de la cause impériale ${ }^{61}$. Quant à l'armée, comme en témoigne clairement la série des inscriptions religieuses de Novae ${ }^{62}$, elle perpétuait, à la faveur des cérémonies collectives et officielles, le culte des dieux du panthéon romain, des empereurs et des symboles de la vie militaire.

Les analyses précédentes, effectuées sur la base d'échantillons et non à l'aide de l'inventaire exhaustif du matériel épigraphique, s'inspirent aussi d'autres données de la vie religieuse dans les provinces à l'époque impériale, à un moment où celles-ci connaissaient des conditions relativement paisibles. Les réflexions concernent principalement les dévotions locales de soldats qui ne sont pas, a priori, directement liés à la divinité remerciée ou honorée, car rien n'indique qu'un culte leur était réservé dans le camp de l'unité à laquelle se rattachait le soldat. La démarche suggère plus nettement que d'autres approches que la nature indigène des divinités n'était en rien un obstacle à des dévotions variées de la part d'individus intégrés à l'empire et que, dans un certain nombre de circonstances et de provinces, ces cultes avaient une fonction comparable à celle attribuée aux cultes orientaux qui n'étaient eux-mêmes que des cultes d'origine provinciale et locale. Le terme de divinité «locale» est, en ce sens, plus satisfaisant que celui d' «indigène» 63 . Aussi, le caractère à tout prendre limité des dédicaces à des divinités désignées comme indigènes se comprend-il par la diffusion géographiquement restreinte de leur culte. Quand un document apparaît voué à une divinité hors de la zone traditionnelle de diffusion, cela s'explique très fréquemment par l'origine du dévot. Le phénomène le plus intéressant est bien celui qui révèle l'attrait exercé par les dieux locaux sur les étrangers de passage et sur les occupants temporaires d'un lieu donné. À titre personnel, le soldat recourait à des forces divines extérieures au

61 G. Alföldy, Die Krise des Imperium Romanum und die Religion Roms, Religion und Gesellschaft in der römischen Kaiserzeit, Cologne, 1989, p. 53-102, met bien en valeur la persistance de la tradition religieuse romaine tant du point de vue des dévotions et des croyances que de la pratique. Il n'y a pas de diffusion nouvelle des cultes indigènes au $\mathrm{III}^{\mathrm{e}}$ siècle.

62 ILNovae, n. ${ }^{\circ} 1$ à 32.

63 Irby-Massie, Religion, p. 212-214, rappelle à juste titre les éléments de rapprochement autant que de différenciation entre les différents types de cultes. 
camp, ce qui ne veut pas dire qu'il excluait les autres. On peut admettre alors que les dédicaces expriment une présence temporaire du militaire quand elles n'indiquent pas clairement son origine ${ }^{64}$; qu'elles dévoilent aussi que les numina installés localement et tutélaires de communautés pérégrines ou non jouissaient d'une efficacité précisément définie, due non à la forme ou à la limitation de leur pouvoir ou de leurs fonctions, mais à leur maîtrise singulière des données locales dans leur ensemble. Celles-ci avaient contribué à leur succès et à l'avènement d'un culte. Le contexte peut seul rendre compte de la nature du transfert ou de l'interprétation effectuée par le dévot ou des modes d'action de l'entité divine objet de la promesse ${ }^{65}$. Cependant, ces aspects ne préjugent pas de la nature du sanctuaire dans lequel le vœu avait pris place. Pour le reste, c'est à travers des formes romaines que les soldats dialoguaient avec ceux dont ils espéraient une faveur ou une attention particulière dans un métier où les déplacements étaient fréquents et où les modes de raisonnement poussaient les professionnels de la guerre à scander les événements chanceux ou heureux d'une carrière par l'offrande d'un autel, d'une statue et, parfois même, d'un sanctuaire.

64 Les auxiliaires semblent plus nombreux que les légionnaires - ce que révèle aussi la documentation bretonne - et on pourrait penser que ce n'est qu'une question de statut. Mais ces auxiliaires sont citoyens romains et les légionnaires d'origine provinciale n'étaient pas tous, loin de là, les descendants de colons italiques.

65 On pourrait s'étonner, s'agissant d'un vœu à une divinité locale que la promesse ait été transférée sur un pouvoir divin qu'ignorait peut-être le soldat au moment de son engagement. C'est sans doute l'indication de liens étroits, à titre personnel entre le dévot et le numen, comme je l'ai dit, mais cela pose aussi la question de la formulation même de la promesse initiale, relativement vague, dans la mesure où un soldat ne pouvait jamais savoir quand et où exactement il aurait à s'acquitter de son dû. L'efficacité recherchée s'accommodait de diverses données et circonstances. 


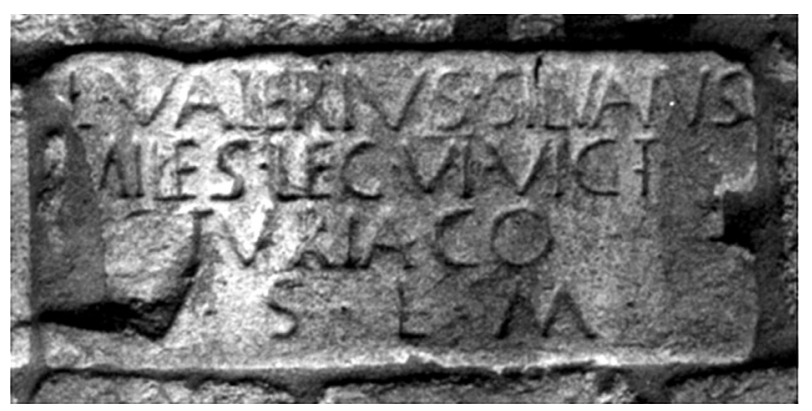

FIG. 1

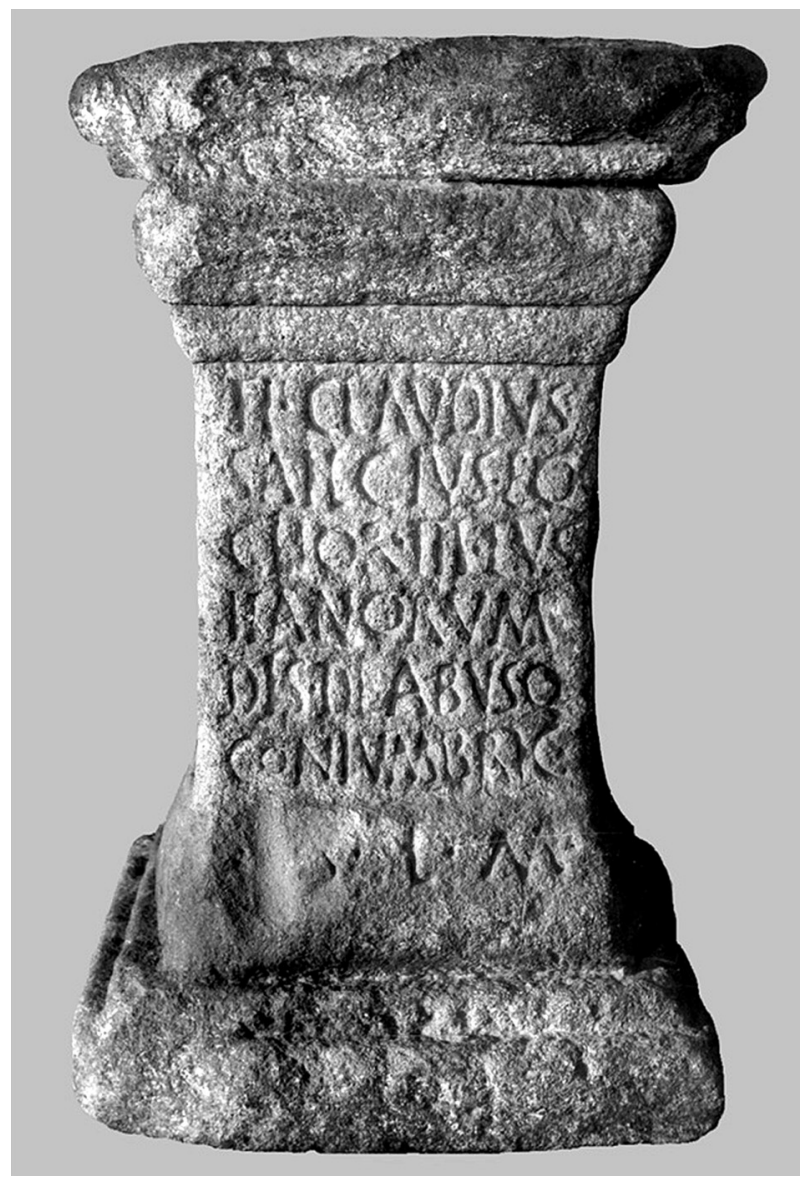

FIG. 2 


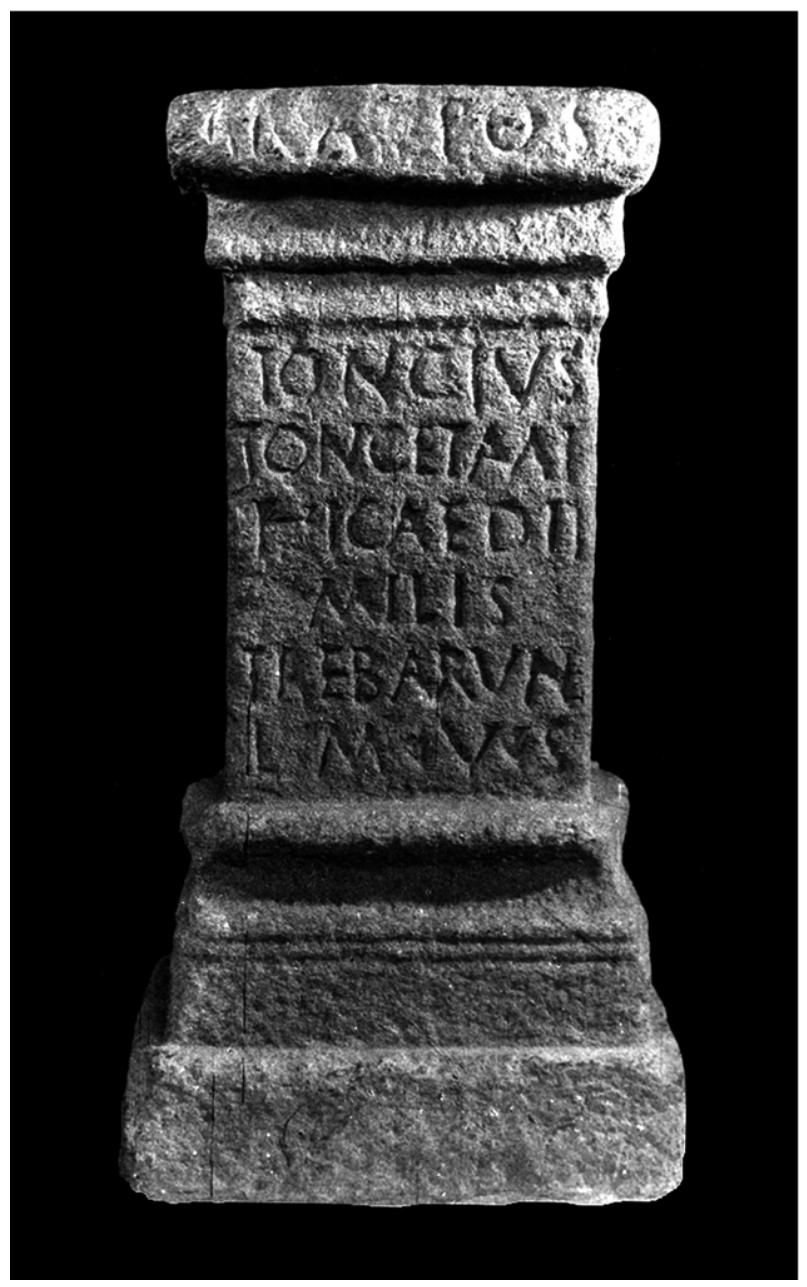

FIG. 3 